\title{
Selection and Customization of Open Source Solutions for Lab Management
}

\author{
Lynette K. Ballast*, Dean Powers** \\ *Cerium Laboratories, LLC, M/S 512, 5204 E. Ben White Blvd., Austin, TX 78749 \\ **Gadoz, Inc., 9017 Latone Ave. NE, Seattle, WA 98115
}

Managing an analytical laboratory and its resources efficiently requires tracking samples as they are routed through the lab. A laboratory information management system (LIMS) is used by many labs to track samples. LIMS also provides the analyst with the information needed to complete the analysis, collect sample results and data, and provide statistical information such as tool utilization, sample turn time and customer sample load to the operations manager. Some LIMS applications can also be used to schedule tool time and resources, and to store information and data related to a particular analysis request.

Cerium Laboratories, LLC is a wholly owned subsidiary of AMD. Cerium Labs originated from AMD's Austin, Texas Process Characterization and Analysis Lab. As Cerium has expanded into the commercial market it has gained many worldwide customers which required that it's LIMS system be accessible to external customers, not just to AMD employees. As a new company, however, there was not a large capital budget, so commercial LIMS applications, which can cost on the order of $\$ 100,000$ and above, were not an option.

Cerium selected Gadoz, Inc. to modify a Zope open source application known as "PloneCollectorNG", which was originally developed to track bugs for software developers. Zope is an Open Source Application and Database, which means that it available free of charge. The advantages of modifying an open source application include no software licensing costs, support from multiple vendors, continual upgrades, and features customized for specific applications. Additional savings can be made by running the Zope application on standard hardware using the Linux operating system. In our examination of commercial LIMS systems we found that most require an Oracle or Microsoft SQL server database and separate server software that significantly increases the cost.

The initial deployment of the LabCollector LIMS has many features to support the Cerium customer base. Work requests are entered via a secure internet site by the customer. The work request information has been customized to fit the services provided by Cerium and the work processes of their engineers and technicians. The data entry is streamlined with as few keystrokes as possible so that entering a large numbers of samples is done easily. Additional information needed to perform the work requested can be securely uploaded by the customer for the analysts to view. Data and reports can be uploaded to the job form for the customer to review via the internet, Fig.1. If time zone differences make it difficult to communicate with the lab personnel directly the customer can enter comments into job requests that are viewable by the lab analyst.

Samples follow a workflow system that is tracked in the LabCollectorNG. This tracking allows the LabCollectorNG to be used as a management tool. When a request moves from one state to another (e.g., "In process" to "Complete") an entry is made in the jobs transcript, Fig.2. For compliance with ISO 17025 all transactions logged against a work request can be tracked. This data that is 
stored can then be mined to provide the operations manager information necessary for planning. Automatic reports can be generated or individual searches can be done at any time.

LIMS applications are vital to manage an operation that processes over ten thousand samples a year. Unfortunately many commercial products are extremely expensive and require additional software and hardware to link them to all the tools in the lab. Modifying an open source application is low cost, requires no additional hardware and can be customized to fit the needs of the lab.

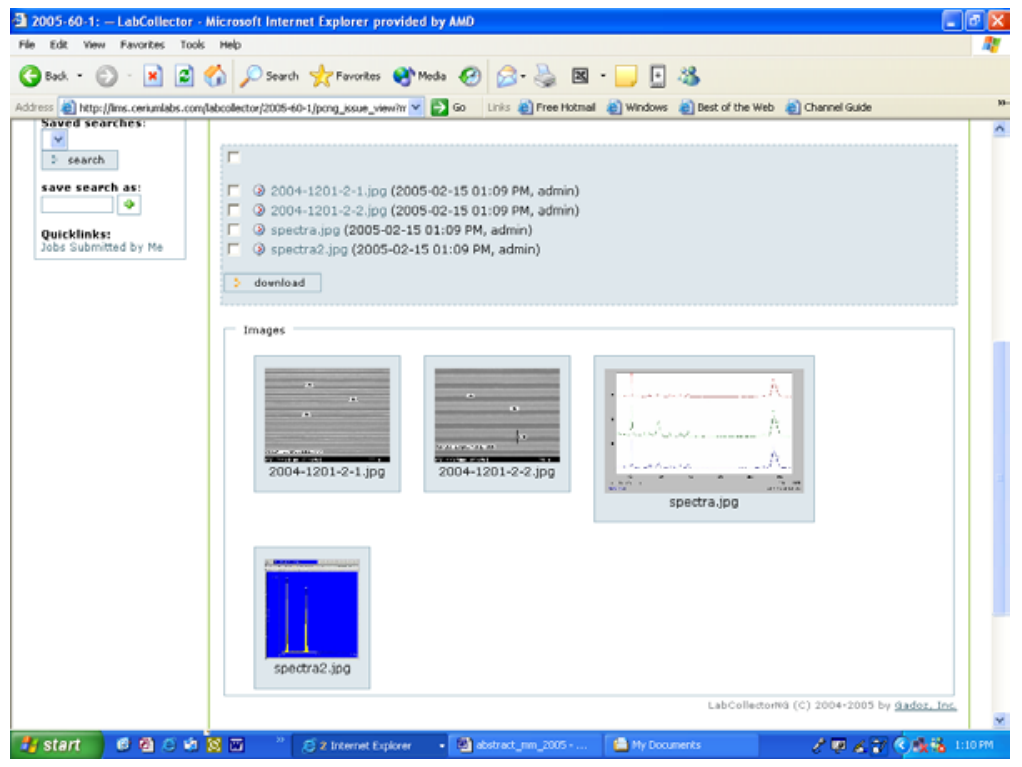

Fig.1. Screen shot of customers image results. Full size images can be viewed by selecting the thumbnail. Images can also be downloaded to the customers local PC.

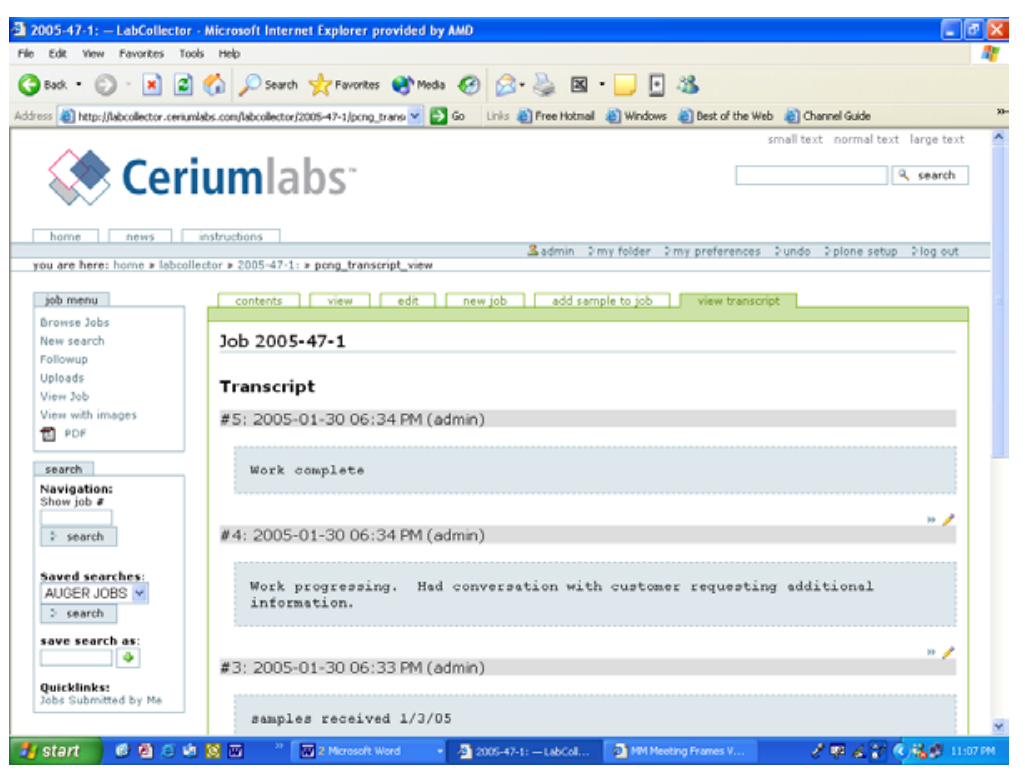

Fig.2. Transcript view of events logged against a selected job request. This feature provides an audit trail for each job including the date, time, and user who performed the action. 